\title{
Anisotropy of colloidal components propels field-activated stirrers and movers
}

\author{
Gabi Steinbach $\odot,{ }^{1,2, *}$ Michael Schreiber $\odot,{ }^{2}$ Dennis Nissen, ${ }^{3}$ Manfred Albrecht, ${ }^{3}$ Sibylle Gemming, ${ }^{1,2}$ and Artur Erbe ${ }^{1}$ \\ ${ }^{1}$ Helmholtz-Zentrum Dresden-Rossendorf, Institute of Ion Beam Physics and Materials Research, \\ Bautzner Landstrasse 400, 01328 Dresden, Germany \\ ${ }^{2}$ Institute of Physics, Technische Universität Chemnitz, 09107 Chemnitz, Germany \\ ${ }^{3}$ Institute of Physics, University of Augsburg, 86159 Augsburg, Germany
}

(Received 13 January 2020; accepted 1 April 2020; published 28 April 2020)

\begin{abstract}
Propulsion via cyclic moves presents a challenge to micro-objects in overdamped environments, where time reversibility may prevent directed motion. Most reported cyclic movers exploit anisotropic hydrodynamic drag with the surrounding medium to push or pull themselves forward. Here, we present a propulsion strategy that exploits internal interactions in multicomponent objects. We study self-assembled clusters of magnetic particles that exhibit an off-center dipole moment. By theoretical modeling and in experiments with magnetic Janus particles, we demonstrate that under an oscillating field the interaction between such anisotropic particles in the cluster breaks time reversibility. This enables propulsion under an oscillating actuation field without changing the external shape of the object, which is impossible within the strategy of hydrodynamic drag. Using the same experimental particle system, we realize various modes of motion ranging from stirrers to steerable movers with helical or directed path under the same environmental conditions. They present a powerful counterpart to propellers that move via hydrodynamic drag.
\end{abstract}

DOI: 10.1103/PhysRevResearch.2.023092

\section{INTRODUCTION}

Locomotion is a fundamental requirement for any form of life to explore their environment. Often, it involves the cyclic repetition of coordinated movement patterns. Locomotion of microscopic objects and organisms moving through a dense medium presents a particular challenge as they face overdamped environments where drag dominates over inertia $[1,2]$. As a consequence of drag, cyclic motion patterns are time reversible [3] - under any oscillating pattern of motion, an object repeatedly goes back and forth by the same distance and lacks directed migration. Yet, nature has found ways for microorganisms to swim [4-8]. To explore the principles, propulsion strategies via cyclic body moves in overdamped environments has been the subject of intense research [9-14]. Moreover, artificial systems have been established using fieldactivated or catalytically active micro-objects [10,15-18].

So far, most reported examples of cyclic movers exploit hydrodynamic coupling via the surrounding viscous medium to convert rotational motion into translation $[18,19]$. For rigid objects, coupling appears if the rotating object has a chiral shape (propeller) [20,21], or if the environment is anisotropic, e.g., the motion takes place close to a surface (roller) [22-26]. Further, multicomponent objects with a flexible tail, resembling flagella (swimmer) [27-29], can perform periodic shape

\footnotetext{
*Corresponding author: gabi.steinbach.de@gmail.com
}

Published by the American Physical Society under the terms of the Creative Commons Attribution 4.0 International license. Further distribution of this work must maintain attribution to the author(s) and the published article's title, journal citation, and DOI. deformations, i.e., undulations, such that hydrodynamic drag leads to translation. These explanations disregard that many discussed objects (whether invariant [20] or flexible [27,30] in object shape) are composed of multiple components, which provides additional flexibility when the components rotate inside the object. However, within the concept of hydrodynamic coupling, internal rotations do not break time reversibility and thus are not essential to realize propulsion.

Here, we present a different actuation concept where internal rotations in multicomponent objects can cause propulsion. The key is that the driven rotation of the components is coupled with an interaction between the components that breaks time reversibility. We study self-assembled clusters of magnetic microparticles. The spherical particles are actuated remotely with an oscillating external field, which causes the magnetic particles to periodically reorient via alignment along the field. This type of actuation is interesting in the context of propulsion principles. To achieve directed migration, at least two coordinates of motion of an object must be time dependent. Trivially, this is the case under a rotating drive, which acts along two coordinates. In contrast, a linearly oscillating drive acts along one coordinate of motion only. However, a coupled interaction between the rotating components can introduce an additional coordinate of motion if the interaction leads to motion along a coordinate that differs from the direction of the field. If this motion is nonreversible, it leads to propulsion.

The magnetic interactions between the particles we employ lead to a nonreciprocal rotation under field actuation. To transform the driven rotation into an effective translation, two conditions must be fulfilled: First, the field-driven rotation creates a force on the center of mass of the particles (i.e., the center of rotation is differs from the center of mass), and 
(a)

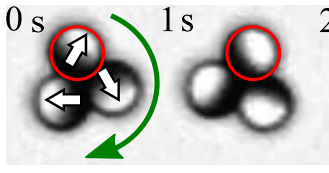

$2 \mathrm{~s}$

(b)

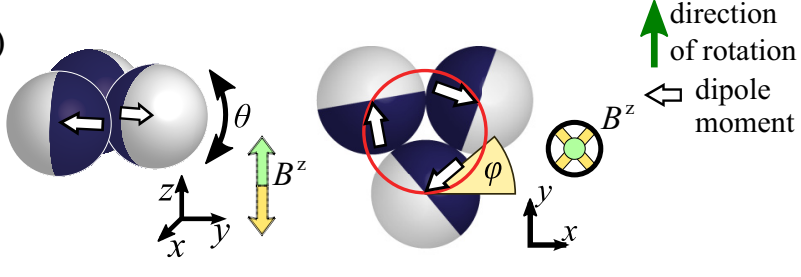

(c)

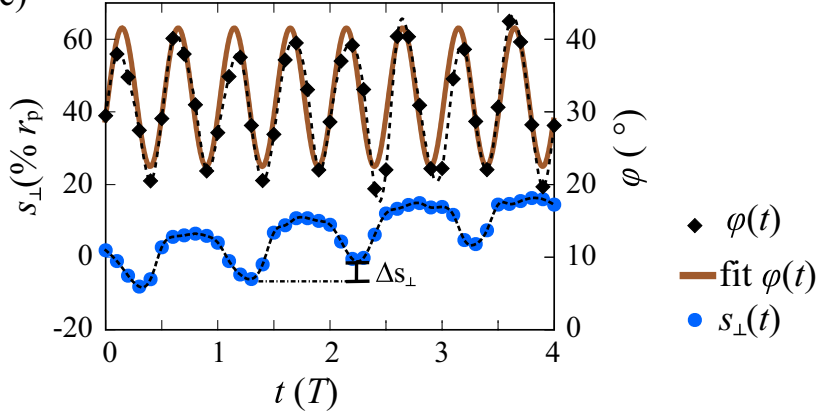

FIG. 1. Experimental actuation of a ring composed of magnetic Janus particles [40]. (a) Image sequence of the rotation of a triple ring under an oscillating field. The red circle of one particle is a guide to the eye for the overall cluster rotation (scale bar $5 \mu \mathrm{m}$ ). (b) Sketches of side and top view of a triple ring, with orientation angles $\varphi$ and $\theta$ of the Janus particles. The magnetic hemisphere is colored in dark blue. Here, the red circle indicates the orbit of in-plane particle motion $\left(s_{\perp}\right)$ spanned by the particle centers. (c) Orientation $\varphi(t)$, the sine fit curve (solid brown), and the orbital translation $s_{\perp}(t)$ of a capped particle in the ring during four periods $T\left(B_{0}^{z}=0.34 \mathrm{mT}, \omega / 2 \pi=2 \mathrm{~Hz}\right)$.

second, the motion pattern of the center of mass becomes nonreciprocal due to the coupled interparticle interaction. We will demonstrate that both conditions can be realized if the cluster is composed of particles with an anisotropic magnetization distribution [31,32].

We employ colloidal particles with a radius of $r_{p}=$ $2.27 \mu \mathrm{m}$, which are equipped with a hemispherical ferromagnetic thin film of $[\mathrm{Co}(0.28 \mathrm{~nm}) / \operatorname{Pd}(0.9 \mathrm{~nm})]_{8}$ [33] to form so-called capped or Janus particles. After magnetic saturation, those particles exhibit a stray field with dipolar characteristic [34], and the dipole moment points along the Janus director. Note that despite the optical similarity, magnetic Janus spheres in previous reports exhibit a dipole that points perpendicular to the Janus director [35-37]. The hemispherical coating causes a negligible mass imbalance, but it provides a significant shift of the magnetic center of more than $0.68 r_{p}$ (Appendix A). In aqueous solution, after sedimentation on the glass substrate the particles assemble in various compact two-dimensional structures with diverse magnetic order $[31,32,38]$. The particle orientation is visualized by transmission light microscopy via the optical contrast between the transparent, uncoated hemisphere and the intransparent, coated hemisphere [Fig. 1(a)]. A set of three pairs of electromagnetic coils is mounted around the sample cell, providing low-frequency actuation fields.
Experimentally, we show that self-assembled clusters move under oscillating fields and that various motion patterns can be realized by different cluster configurations. We then explain the actuation concept qualitatively with a simple theoretical model. It describes the motion of spheres with an embedded dipole that is not centered but shifted radially outwards, so-called shifted-dipole particles (sd particles) [39]. The key point is that the interaction between spheres with this magnetic asymmetry breaks time-reversal symmetry under oscillating fields. The findings demonstrate that internal rotations in multicomponent objects built from anisotropic particles enable new actuation strategies for cyclic movers that complement established schemes based on hydrodynamic drag.

\section{RESULTS}

\section{A. Experimental findings}

Among other possible configurations, the magnetic Janus particles spontaneously form clusters of three particles, with the dipole moments forming a flux closure ring [Fig. 1(a)] [32]. We apply an oscillating magnetic field $B^{z}$ perpendicular to the assembly plane of the triple ring [see sketch in Fig. 1(b)]. The oscillating field drives a synchronous up and down oscillation of the Janus particles by angle $\theta$ [Fig. 1(b), left]. As the cluster is close to a glass substrate, hydrodynamic coupling causes the rotating particles to repeatedly roll back and forth. For single particles, this translation is reversible, which excludes directed motion. However, for Janus particles that form as a ring, we observe an overall rotation of the ring around an axis parallel to the field. During the rotation, the uncapped, nonmagnetic hemispheres always faces towards the rotation direction [Fig. 1(a)] [40].

Environmental conditions such as the chemical difference across the particle surface or the magnetic field can be excluded as possible reasons for the symmetry breaking (Appendix B). Further, structural deformations of the cluster can be excluded; the cluster shape remains constant even after many field cycles (Appendix C). In order to scrutinize the ring rotation, we have analyzed the translational and rotational motion of the particles in the triple ring in more detail using image analysis. During cluster rotation, the particles effectively translate on an orbit spanned by the particle centers [see red ring in Fig. 1(b), right]. This translation of the particles perpendicular to the field is shown as $s_{\perp}(t)$ in Fig. 1(c). The position oscillates with a periodicity that matches the applied field frequency. This visualizes the substrate-induced back and forth rolling. Remarkably, the oscillation is not a symmetric sine curve. The hills are much more oblate than the valleys, with a ratio of the amplitudes of about $\approx-0.2$. Moreover, the graph $s_{\perp}(t)$ exhibits a gradual increase. During each field cycle, a particle moves by $\Delta s_{\perp}$, which leads to the overall ring rotation. Besides the translation, we analyze the azimuthal orientation of the particle (Janus director projected onto the assembly plane). We define the azimuthal angle $\varphi$ that is enclosed by the in-plane component of the Janus director and the tangent to the orbit of motion [Fig. 1(b), right]. We find that the orientation of $\varphi(t)$ changes during field application [Fig. 1(c)]. It oscillates periodically with a 
frequency twice the field frequency of $2 \mathrm{~Hz}$. Moreover, the value $\varphi=32^{\circ}$ which the particles obtain when $\theta=0$ during the rotation is different from the equilibrium configuration of three particles in the absence of external fields, which is characterized by $\varphi=50^{\circ}$ [32]. These steady-state oscillations [Fig. 1(c)] are consistent with our previous findings for twoparticle clusters, which also obtain a nonequilibrium steady state under oscillating fields [31].

This in-plane oscillation of $\varphi(t)$ with doubled frequency, which is clearly not induced only by the external field, breaks time reversibility of the rotational motion of the particles: The azimuthal orientations $\varphi$ during out of-plane rotation $(|\theta|$ increases) differ from the orientations when the particles rotate back into plane $(|\theta|$ decreases). We propose that this leads to the overall ring rotation. As we excluded all possible external causes, the time dependence of $\varphi(t)$ must result from the magnetic interparticle interaction.

Before presenting a comprehensive numerical explanation for the ring rotation based on the motion $s_{\perp}(t)$ in Fig. 1(c), we want to demonstrate that the field-driven actuation can result in a variety of motion paths that depends on the internal cluster configuration. We exploit the fact that the Janus particles studied here self-assemble into compact clusters with various magnetic configurations $[38,39,41]$. Essentially, self-assembling Janus particles form clusters with nonlinear dipole configuration to minimize the magnetic interaction potential. A bistability in the interaction potential [32] leads to variability in the magnetic configuration of a given cluster. As an example, clusters consisting of five particles are discussed here. While there exists only one possible spatial shape for a compact five-particle cluster, it can appear in different magnetic configurations (Appendix D). Two of the possible configurations are shown in Figs. 2(a) and 2(b). They differ in the in-plane orientation of only one particle [leftmost in the insets in Figs. 2(a) and 2(b)] while all other four particles are similar. This seemingly small difference in the magnetic configuration leads to a drastic difference in the trajectory of the cluster when actuated by oscillating fields [Figs. 2(a) and 2(b)]. Configuration (a) performs a helical motion while the other, (b), exhibits an almost linear path [42]. The center of both configurations moves with about the same speed (about $0.1 \frac{\mu \mathrm{m}}{\mathrm{s}}$ ), but the superimposed circular and lateral motion of the helical mover (a) leads to an effectively slower lateral translation of about $0.04 \frac{\mu \mathrm{m}}{\mathrm{s}}$.

Assuming that each particle in a cluster contributes with $s_{\perp}$ along the in-plane orientation of its cap, the motion path of a cluster arises approximately from the sum of the dipole vectors. In configuration (a), the dipole vectors of the particles form a bent ring. In configuration (b), the vector sum of the cap orientations gives a significant cluster moment, which leads to a more directed translation (see Appendix D for more details). Moreover, the residual cluster moment of the latter can be used to orient that cluster and steer translation in a superimposed, constant in-plane field $B^{x, y}$, provided that the field intensity is too low to alter the cluster structure. When orienting the cluster with an additional constant planar field, it performs directed translation under the oscillating perpendicular field $B^{z}$ [Fig. 2(c)]. Switching from $B^{y}$ to $B^{x}$ changes the translation direction by $90^{\circ}$ [43]. It is noteworthy that the translation path in Fig. 2(c) deviates from the direction
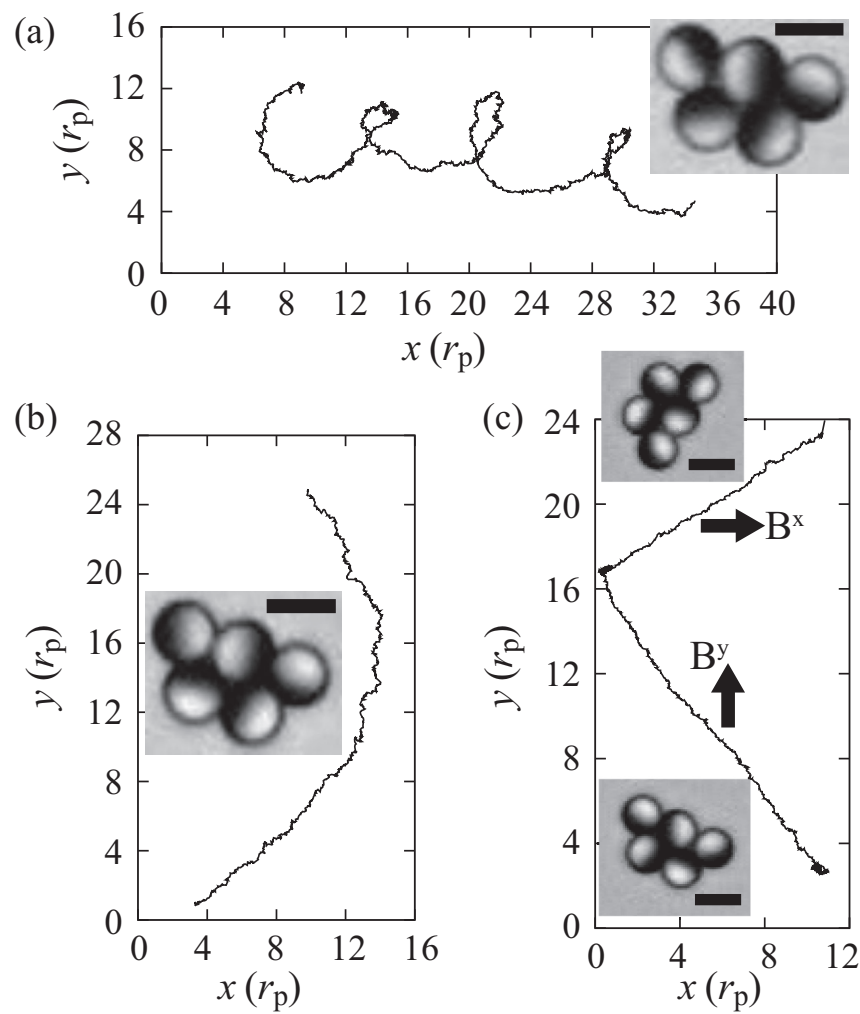

FIG. 2. Experimental trajectory of two compact clusters (insets) (a), (b) under an oscillating field with $B^{z}=0.53 \mathrm{mT}, \omega / 2 \pi=$ $5 \mathrm{~Hz}$ (a: $88 \mathrm{~s}$; b: $251 \mathrm{~s}$ ) [42] and (c) under an oscillating field $\left(B^{z}=1.13 \mathrm{mT}, \omega / 2 \pi=20 \mathrm{~Hz}\right)$ and a constant in-plane field $\left(B^{x, y}=\right.$ $0.03 \mathrm{mT}$ ) [43]. The in-plane field is switched from $B^{y}$ to $B^{x}$ during the recording (scale bars $5 \mu \mathrm{m}$ ).

of the applied field, because the net magnetic orientation of this cluster does not coincide with its effective translation direction.

\section{B. Numerical model of the actuation}

For the theoretical description of the observed motion of the colloidal cluster, we employ the conceptually simple sd-particle model: spheres with an embedded dipole that is shifted radially outwards. This model has been used earlier to successfully reproduce the assembly and rotational dynamics of the magnetically anisotropic Janus particles [31,32]. The explanation of the actuation process requires three steps, which are also demonstrated visually in Figs. 3(a)-3(c). First, the conversion of field-driven rotation into time-reversible displacement is achieved by each anisotropic sd particle separately [Fig. 3(a)] and hence is an effective single-particle problem. Second, when sd particles are spatially arranged in a cluster they mutually hinder their field-induced single-particle displacement [Fig. 3(b)]. This impacts magnitude and direction of the displacement, which is taken into account by introducing an angle-dependent hindrance factor $c_{h}$. Third, when particles in a cluster perform synchronous, field-driven outof-plane oscillation, they exhibit an additional in-plane oscillation that emerges from the magnetic interaction between the particles [Fig. 3(c)]. This collective in-plane oscillation 
(a)

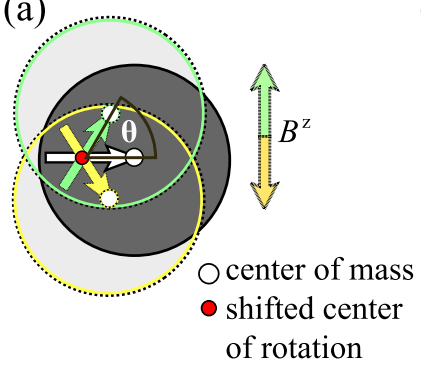

(b)

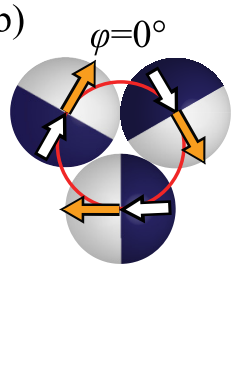

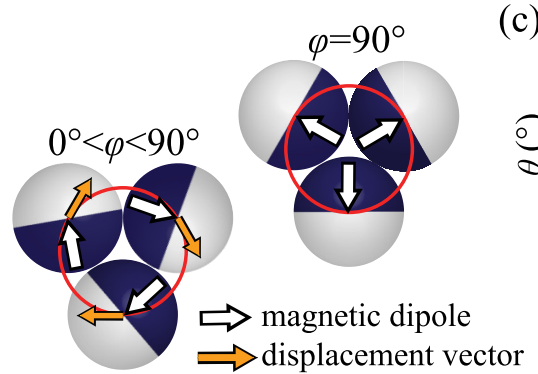

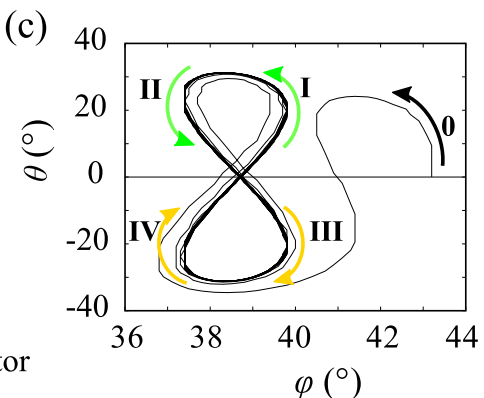

(d)
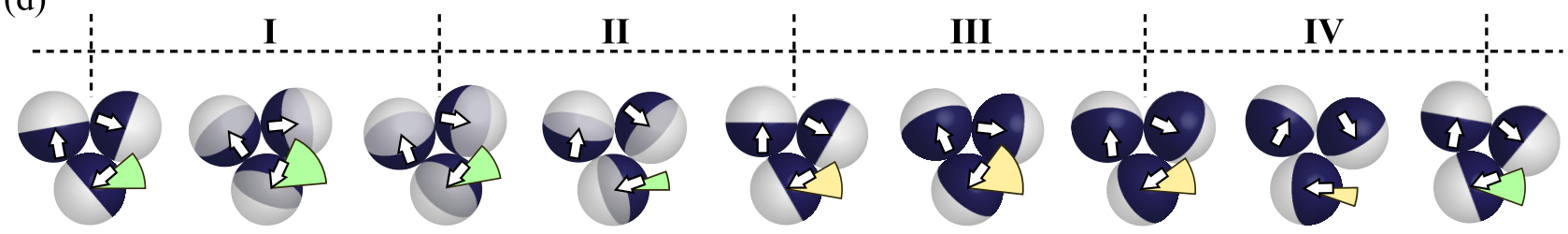

FIG. 3. Numerical model of the actuation principle using a triple ring of sd particles. (a) Sketched side view of the off-centered rotation of an sd particle under an oscillating field $B^{z}$. (b) Sketch of steric hindrance between contacting particles in a triple ring. When an oscillating field $B^{z}$ is applied, the sd particles are forced to move along the in-plane component of the dipole vector, but steric hindrance between particles allows displacement only tangentially to the ring. Thus displacement is unobstructed only for $\varphi=0^{\circ}$, decreases for $\varphi<90^{\circ}$, and is completely obstructed for $\varphi=90^{\circ}$. (c) Numerically calculated trajectory in $\theta-\varphi$ coordinates of sd particles $\left(\xi_{d}=0.55\right)$ in a triple ring under an oscillating field $\left(B_{0}^{z}=\frac{3 \mu_{0} m}{16 \pi r_{p}^{3}}\right)$. The arrows indicate the time evolution. (d) Sketched top view of a triple ring during one movement cycle I $\rightarrow$ IV. For clarity, the variations in $\varphi$ (colored segments) are 20 times larger than the ones plotted in (b). The colors of the segments correspond to the colors of arrows in (c). (See also [46] for an animated illustration.)

is nonreversible, and in combination with the displacement it causes an effective motion of the whole cluster [Fig. 3(d)]. In the following, these three steps are explained in detail.

First, the periodic single-particle displacement under an oscillating field is a consequence of the magnetic anisotropy of the sd particles [Fig. 3(a)]. An oscillating field exerts a torque on the magnetic center of a dipolar particle, and the particle follows the field by periodically rotating up and down by angle $\theta$. In the case of sd particles, where the magnetic center is shifted away from the center of mass, the applied field consequently displaces the center of mass of the sd particles along an arc [44] [Fig. 3(a)]. For particles surrounded by a medium, dissipative interactions cause only a slight modification of the rotation center (Appendix E). Without loss of generality, we can assume that the particle rotates around a center that is shifted by the amount $\xi r_{p}$ from the center of mass of the sphere with radius $r_{p}$. An additional influence arises when our particles rotate in the vicinity of a substrate. Wall-induced hydrodynamic drag causes additional conversion of rotation into translation. Here, this conversion is not constant, since the off-center rotation involves a periodically changing distance between particles and the substrate. (For more details, see Appendix F.) To approximate the influence of this oscillating sphere-substrate interaction, we choose a $\theta$ dependent conversion factor $c_{1,2}$, which assumes the constant value $c_{1}$ while the cap points up $(\theta<0)$ and $c_{2}$ while the cap points down $(\theta>0)$, with $c_{1}>c_{2}$. We, however, emphasize that the impact of the surface on the motion does not break time reversibility; a single oscillating sphere near the surface does not perform effective translation.

For a single sd particle, we can derive an analytic expression for the infinitesimal in-plane displacement $\mathrm{d} s_{\perp}$ of the center of mass that ideally arises from the rotation by an angle $\mathrm{d} \theta$ around the rotation center as

$$
\mathrm{d} s_{\perp}=-\xi r_{p} c_{1,2}(\theta) \sin \theta \mathrm{d} \theta .
$$

Integration over a whole field cycle gives the overall translation. It vanishes for a single particle that is not bound to a cluster; it thus undergoes reversible oscillations as shown in [45]. (For a discussion of the motion $\mathrm{d} s_{\|}$parallel to the applied field, see Appendix F.)

Next, we consider sd particles that are assembled in a ring cluster. We concentrate on the triple ring as the smallest, highly symmetric entity showing the actuation effect. In analogy to a single sd particle, field-induced rotation by $\mathrm{d} \theta$ displaces each sd particle by $\mathrm{d} s_{\perp}$. However, in contrast to a single particle, the particles in a cluster cannot move freely and their displacement is hindered by the presence of other particles as described next. The displacement of particles in a ring is confined to the orbit spanned by the particle centers [red ring in Fig. 3(b)], with the orbit being fix due to the mutual magnetic attraction between the particles. In other words, the in-plane displacement vector of a particle in the ring always points along the orbit tangent, indicated by yellow arrows in Fig. 3(b). Consequently, the dipole vector and the displacement vector do not align if the dipole does not align tangentially to the orbit, i.e., $0^{\circ}<\varphi<90^{\circ}$ [Fig. 3(b)]. While the field-driven rotation of sd particles forces them to displace along the in-plane direction of the dipole vector, particles are sterically hindered by the presence of other particles in contact. For synchronously rotating particles, the displacement $\mathrm{d} s_{\perp}$ is completely unobstructed only if $\varphi=0^{\circ}$, i.e., the displacement vector aligns with the dipole vector [Fig. 3(b)], and $\mathrm{d} s_{\perp}$ decreases with decreasing values of $\varphi$ until it reaches $\mathrm{d} s_{\perp}=0$ when the dipoles point towards the center of the orbit [case of $\varphi=90^{\circ}$ in Fig. 1(b)]. We can 
take this anisotropic, angle-dependant hindrance into account by an additional factor $c_{h}(\theta)=\cos \varphi(\theta)$. Within this approximation, the hindrance between interacting particles depends exclusively on $\varphi$. In the experimental study above, we have already shown that $\varphi$ is an oscillating function of $\mathbf{B}^{z}$. This gives rise to a time-irreversible interparticle hindrance term $c_{h}(\theta)$.

Finally, to determine $\varphi(t)$ of sd particles in a triple ring, we have studied the rotational (angular) motion of the particles separately from their translational one. We numerically solve the equation of rotational motion of interacting sd particles in the ring under an oscillating field $\mathbf{B}^{z}=\mathbf{B}_{0}^{z} \sin (\omega t)$ (Appendix G). We apply a dipole shift of $0.55 r_{p}$, which describes the experimental particles well $[31,32,39]$. As we have shown previously, for two interacting sd particles in an oscillating external field $\mathbf{B}^{z}$, the dipoles follow doubleloop trajectories in $\theta-\varphi$ coordinates [32]. The same type of trajectory is observed for particles in larger assemblies [Fig. 3(c)]. After a short transient oscillation (0), the dipoles reach a steady-state loop along the path I $\rightarrow$ IV. The double loop is a very small effect, as the variations in $\varphi$ and $\theta$ differ by one order of magnitude. The character of the angular motion can be visualized more easily with amplified variations in $\varphi$ [Fig. 3(d)] [46]. The dipoles obtain larger angles $\varphi$ while rotating out of plane (increasing $|\theta|$ in I, III) than during the rotation back into plane (decreasing $|\theta|$ in II, IV). The loop trajectory $\varphi(\theta(t))$ breaks time reversibility, since the sense of direction of the loop is uniquely defined by the chirality of the cluster. Essentially, this nonreversible rotation combined with $\varphi$-dependent displacement of the sd particles leads to directed motion of a particle cluster as demonstrated in the following.

During the increase of $|\theta|$ [small hindrance values $c_{h}(\theta)=$ $\cos \varphi(\theta)]$, the displacement $\mathrm{d} s_{\perp}$ is hindered more strongly than during the decrease of $|\theta|$ [large values $\cos \varphi(\theta)$ ]. For a quantitative analysis of the overall translation, we fit the trajectory $\varphi(\theta(t))$ in Fig. 3(c) to Lissajous curves and insert the functional term together with the anisotropic hindrance term $c_{h}(\theta(t))=\cos \varphi(\theta(t))$ in Eq. (1) (see Appendix $\mathrm{H}$ for details). The overall translation follows from the integration $s\left(t^{\prime}\right)=\int_{0}^{t^{\prime}} \mathrm{d} s(t)$ (Fig. 4). After each field cycle, each sd particle in the triple ring obtains an overall traveled distance $\Delta s_{\perp}$ (inset in Fig. 4). The synchronous motion of the composing particles results in an effective rotation of the whole triple ring [46]. This numerical analysis shows that the directed propulsion in a linearly oscillating external field is solely enabled by the internal magnetic and steric interparticle interactions, which give rise to time-irreversible, anisotropic hindrance conditions. Hydrodynamic interactions, e.g., in the presence of a substrate as given by the factor $c_{1,2}$, are fully reversible and modify the displacements only quantitatively.

\section{Comparison of simulated and measured trajectories}

The numerically obtained translation curve of sd particles qualitatively agrees well with the curve we measured for experimental Janus particles (Fig. 4). Both show an effective translation after each field cycle, and the curves obtain similar shapes. The numerical trajectory $s_{\perp}(t)$ qualitatively equals the experimental findings, even in the sense of rotation; the nonmagnetic hemispheres point in the direction of the overall

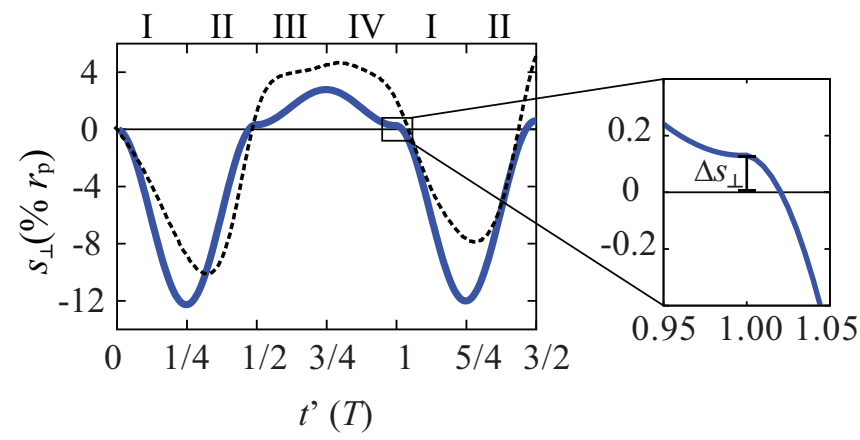

FIG. 4. Translation of numerical sd particles under an oscillating field. The solid curve shows the translation $s_{\perp}$ that each particle in a triple ring performs over time $t^{\prime}$ under an oscillating field, indicating an effective translation $\Delta s_{\perp}$ that particles have obtained after one field cycle $\mathrm{T}$ (see magnified cutout). The curve qualitatively agrees with the curve obtained for experimental Janus particles [dashed curve, taken from Fig. 1(c)]. Roman numbers correspond to the labels in Fig. 3(c).

rotation. For a better quantitative comparison of numerical and experimental findings, in the simulation we applied a substrate-induced conversion factors of $c_{2} / c_{1} \approx-0.2$. This factor has been extracted from the experimental translation curve $s_{\perp}(t)$ in Fig. 1(c) as the ratio between the amplitudes during the section of forward translation (III/IV) and the section of backward translation (I/II).

Despite the qualitative coincidence, the experimental and numerical findings differ quantitatively in $\Delta s_{\perp}$ (Fig. 4). This leads to significant differences in the rotation efficiency of the ring. The experimental triple rings rotate by about $3^{\circ}-10^{\circ} \frac{1}{\mathrm{~s}}$ with a mean relative increase of $\Delta s_{\perp} / r_{p}=3.6 \%$ per cycle. This is one order of magnitude larger than $\Delta s_{\perp} / r_{p}$ in the simulation (Fig. 4). In contrast to the sd particles with a single point dipole, the capped particles provide an extended magnetization distribution. This leads to a quantitative modification of the dipole interaction potential $[32,47]$. The difference affects the radial oscillations $\varphi(t)$, which are a direct consequence of the magnetic interaction between the particles. Fitting the experimental curve $\varphi(t)$ [Fig. 1(c)] to a sine function gives a mean value of $\varphi=32^{\circ}$ and a radial oscillation amplitude of $10^{\circ}$. This radial amplitude in $\varphi$ of capped particles is about 8 times larger than for sd particles. Therefore, also the doubleloop trajectory of capped particles is broadened by this factor. Since the area enclosed by the double loop is proportional to the propulsion efficiency $\Delta s_{\perp} / r_{p}$, it is consistent that the experimental value of $\Delta s / r_{p}$ is also one order of magnitude larger than in the simulation.

Finally, in analogy to the experimental setting, the numerical analysis incorporates the proximity of the cluster to a nearby substrate. Yet, this asymmetry in the environment does not present a necessary prerequisite for the motion; time reversibility is broken only by the magnetic interaction between the anisotropic particles. As a consequence of this concept, the field-actuated clusters should also move if they are located in isotropic environments (bulk fluids). In fact, numerical simulations show that the triple ring effectively rotates also if we assume conversion factors of $c_{1}=c_{2}=1$ (Appendix $\mathrm{H}$ ). In the presented model of sd particles, the 
lack of surface-induced translation only reduces the overall efficiency of the ring rotation.

\section{DISCUSSION}

In conclusion, we have demonstrated experimentally and theoretically that field-driven internal rotations in multicomponent objects play a crucial role for directed locomotion of cyclic movers in dissipative environments. The rotations enable migration under conditions that make locomotion impossible within the usually considered concept of hydrodynamic drag. The propulsion strategy introduced here requires two ingredients. First, the composing components can rotate individually in response to an external field. Second, they must exhibit an anisotropic magnetization distribution. The anisotropic magnetization distribution causes periodic displacement of the composing particles. In addition, the magnetic interaction between such anisotropic components activates torsional oscillations perpendicular to the applied field direction, which breaks time-reversal symmetry. Combining the displacement with nonreversible rotation leads to an effective motion of the multicomponent object.

The actuation concept combines essential benefits. First and most striking, we have shown that it enables a wide variety of motion paths ranging from rotation to helical motion and steerable translation. The motion pattern of any cluster with a given shape is determined by the internal interactions and as a consequence can be reprogrammed by modifying the magnetic arrangement of a cluster without changing the cluster shape. Second, the translation direction can be controlled with an additional external field by steering the overall magnetic moment of a cluster. Finally, the actuation does not require any fuel molecules around the particles; thus the presented system can be fully biocompatible, potentially enabling cargo delivery in living systems. The achievable actuation efficiency of the presented strategy is comparable to those of swimmers that move by anisotropic drag. Reported clusters rotate with about $1 / 10$ of the actuation frequency [48], while we reach an efficiency of about $1 / 20$. Also, our translation speeds of 0.01-5 $\mu \mathrm{m} / \mathrm{s}$ compare with other swimmers, which typically move with a few $\mu \mathrm{m} / \mathrm{s}$ [10].

We suggest that this actuation strategy has valuable implications on other particulate soft matter. Since the motion path crucially depends on the spatial and magnetic cluster configuration, any combination of rotation and translation should be realizable by proper choice of asymmetric particles and assembly of a cluster. We expect even more diverse motion paths when using other magnetically anisotropic particles, which are currently widely discussed for complex selfassembly $[19,35,36,44,49-52]$. Note that for a rigorous theoretical description of the exact translation path, additionally, the hydrodynamic drag of asymmetric clusters must be taken into consideration, which provides a complex problem on its own [53].

Considering the simplicity of the oscillating field pattern that drives the motion, the question remains whether similar actuation can be found elsewhere, e.g., in living systems. Since the propulsion strategy relies on the anisotropy of the particles, an increase of the anisotropy would probably increase the efficiency of the driving mechanism. In addition to anisotropic potentials, also entropic interactions between components with anisotropic shape [54] might enable similar propulsion mechanisms based on the presented strategy of internal rotations.

\section{ACKNOWLEDGMENTS}

Financial support by the German Research Foundation (DFG, Grants No. ER 341/9-1, No. AL618/11-1, and No. FOR 1713 GE 1202/9-1) is gratefully acknowledged. We are thankful to Bjoergvin Hjörvarsson for pointing to the rotating clusters in the microscopy recordings. We thank Henrique Moyses for fruitful discussions.

\section{APPENDIX A: ANISOTROPY OF CAPPED PARTICLES}

The hemispherical coating of the studied silica spheres theoretically leads to shape anisotropy, mass imbalance, and magnetic anisotropy. The extent of the symmetry break for each aspect—-shape, mass, and magnetic center-are detailed next. We have deposited a metallic film with a maximum thickness of $t_{0}=16.5 \mathrm{~nm}$ onto an array of silica spheres with a radius of $r_{p}=2.27 \mu \mathrm{m}$. Deposition normal to the array leads to a hemispherical coverage of each particle. Across the coated hemisphere, the thickness $t$ of the film varies as $t_{0} \cos \Theta$, with the angle $\Theta$ measured with respect to the deposition direction, which is equal to the Janus director. The film deposition leads to a maximum variation of the particle radius of $t_{0} /\left(r_{p}\right)=0.0072<1 \%$, justifying our assumption that capped particles are still spherical.

To calculate the center of mass of the coated particles, additionally, the different volume and mass densities $\rho$ of the silica sphere and of the metal film have to be regarded. The silica spheres have a density of $\rho_{\mathrm{Si}}=2 \frac{\mathrm{g}}{\mathrm{cm}^{3}}$. For the metal film, we estimate a mean density of $\rho_{\mathrm{F}}=12.4 \frac{\mathrm{g}}{\mathrm{cm}^{3}}$, which follows from the densities of each element in the film (Ta: 16.6 $\left.\frac{\mathrm{g}}{\mathrm{cm}^{3}} ; \mathrm{Co}: 8.9 \frac{\mathrm{g}}{\mathrm{cm}^{3}} ; \mathrm{Pd}: 12.0 \frac{\mathrm{g}}{\mathrm{cm}^{3}}\right)$ and their relative fraction of the total film thickness (Ta: $18 \%$; Co: $13 \%$; Pd: $69 \%$ ). The volume of the silica particle amounts to about $V_{\mathrm{Si}}=$ $\frac{4}{3} \pi r_{p}^{3}=4.90 \times 10^{-16} \mathrm{~m}^{3}$. For the volume of the magnetic metal cap, $V_{F}$, we choose the amount of metallic film which is deposited on the substrate area covered by a particle, $V_{F}=$ $\pi r_{p}^{2} t_{0}=2.62 \times 10^{-19} \mathrm{~m}^{3}$. This choice of $V_{F}$ assumes normal incidence conditions during the metal deposition and an ideal sticking of the deposited atoms to the curved silica surface. With these volumes the masses of the two components amount to $M_{\mathrm{Si}}=9.80 \times 10^{-11} \mathrm{~g}$ for the particle and $M_{F}=3.31 \times$ $10^{-12} \mathrm{~g}$ for the cap.

Due to the rotational symmetry of the coated particle, the centers of mass of the silica sphere, $z_{\mathrm{Si}}$, of the magnetic cap, $z_{F}$, and thus also of the complete Janus particle, $z_{\mathrm{M}}$, are located on the symmetry axis. For the following discussion, that axis will be chosen as the $z$ axis, and the geometric center of the silica sphere is defined to be located at $z_{\mathrm{Si}}=0$. In general, the center of mass, $z_{M}$, of an arbitrarily shaped object can be calculated by

$$
z_{M}=\frac{\int d V z \rho}{\int d V \rho},
$$


where $z_{F}$ is obtained by integrating (A1) over the cap in spherical coordinates with polar angle $\Theta$ and azimuthal angle $\phi$. In spherical coordinates, the $z$ coordinate is related the radius $r_{p}$ of the particle and the polar angle $\Theta$ as $z=r_{p} \cos \Theta$. For the hemispherical cap we use $\Theta \in\left[0: \frac{\pi}{2}\right], \phi \in[0: 2 \pi]$, and $r \in\left[r_{p}: r_{p}+t_{0} \cos \Theta\right]$. With a film thickness of $t_{0} \cos \Theta$ at a height of $r_{p} \cos \Theta$, this gives

$$
\begin{aligned}
\frac{1}{V_{F}} \int_{\text {cap }} d V z= & \frac{1}{\pi r_{p}^{2} t_{0}} \int_{0}^{2 \pi} d \phi \int_{0}^{\frac{\pi}{2}} r_{p} \sin \Theta \cos \Theta d \Theta \\
& \times \int_{r_{p}}^{r_{p}+t_{0} \cos \Theta} r^{2} d r .
\end{aligned}
$$

This is $\frac{2}{3} r_{p}+\frac{1}{2} t_{0}$ to the first order in $t_{0}$ and amounts to $z_{F}=1.52 \times 10^{-6} \mathrm{~m}$. From $z_{F}$ and $z_{\mathrm{Si}}$ and the masses $M_{\mathrm{Si}}$ and $M_{F}$ one finds the mass center for the Janus particle at $z_{M}=5 \times 10^{-8} \mathrm{~m}$ shifted away from the center of the sphere towards the cap. This corresponds to $2.2 \%$ of $r_{p}$. This implies that the overall shape and mass distribution of a capped particle deviates only negligibly from those of an ideal sphere, and the mass imbalance can be neglected here.

Next we will estimate the dipole shift of the capped particles. The magnetization distribution of the capped part is radially symmetric (dipolelike), and the magnetic center is displaced along the symmetry axis away from the particle center towards the cap. The mass center of the cap at $z_{F}=$ $1.58 \times 10^{-6} \mathrm{~m}$, or $68 \%$ of $r_{p}$, is a natural first estimate for the magnetic center, assuming that the film exhibits an outof-plane magnetization. Yet, thin parts of the cap may become superparamagnetic and thus may not contribute, while the top part of the cap will dominate the net magnetization [34]. Hence, $z_{F}$ represents only a lower bound for the actual location of the magnetic center along the symmetry axis. $\mathrm{Nu}-$ merical simulations of small clusters assembled from capped particles suggest that the effective shift is in the range between $60 \%$ and $78 \%$ of $r_{p}$ [32]; this range includes the estimate above.

\section{APPENDIX B: EXCLUSION OF HEMISPHERE SURFACE EFFECTS}

In order to exclude an influence based on the two chemically different (capped and uncapped) surfaces of the Janus particles, we have examined an additional set of particles where an additional layer of silicon oxide $\left(\mathrm{Si}_{3} \mathrm{~N}_{4}\right)$ is deposited on top of the magnetic film. This layer is chemically similar to the uncoated silica hemisphere. Under same experimental conditions, triple rings of both types of particles, with metal and with a silicon oxide top layer, exhibit the same angular speed when exposed to an oscillating field; thus effects related to different surface materials can be excluded.

\section{APPENDIX C: SHAPE STABILITY OF MOVING CLUSTERS}

To prove the assumption that the shape of the clusters does not change over time while an oscillating field is applied, we measure the perimeter of the whole cluster over 60 field cycles (Fig. 5). Any reconfiguration in particle arrangement or deviation from a compact particle assembly causes an immediate change in the cluster perimeter. In all three presented

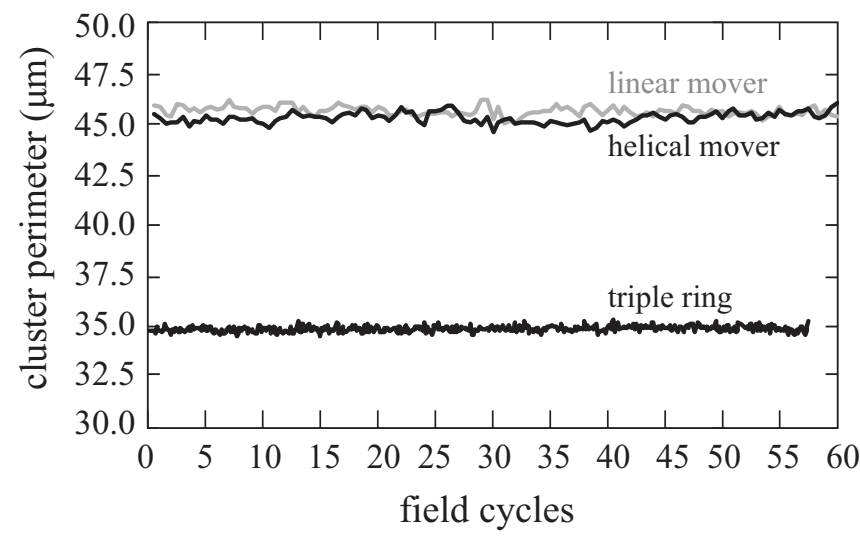

FIG. 5. Shape invariability of clusters under oscillating fields. As a measure for cluster shape, we analyze the perimeter of clusters under field application over time. The perimeter is shown for the rotating triple ring (bottom black curve) (as presented in Fig. 1) and for compact five-particle clusters [Fig. 2(a)] while performing helical motion (top black curve) or linear translation (gray curve). In all cases, the perimeter and thus the shape of the clusters does not change over time.

cases - the triple ring (Fig. 1), the five-particle helical mover [Fig. 2(a)], and the linear mover [Fig. 2(b)] - the perimeter of the cluster remains constant within measurement accuracy. In particular, the perimeter of the triple ring has a perimeter of $34.85 \pm 0.14 \mu \mathrm{m}$. The five-particle cluster provides a constant perimeter independent of the motion path; the helical mover has a perimeter of $45.16 \pm 0.28 \mu \mathrm{m}$, and the linear mover provides $45.55 \pm 0.28 \mu \mathrm{m}$, which coincides well. Assuming that the colloidal particles pack densely, the theoretical perimeters for the cluster are $35.6 \mu \mathrm{m}$ for the triple ring and $49.9 \mu \mathrm{m}$ for the five-particle cluster. Those values agree with the perimeters measured experimentally (Fig. 5), with slight deviations occurring from optical errors. Optical diffraction partially obstructs the areas where two neighboring particles contact each other. This decreases the measured perimeter in comparison with theoretical values.

\section{APPENDIX D: MAGNETIC CONFIGURATIONS IN COMPACT CLUSTERS OF FIVE PARTICLES}

Clusters of capped particles with magnetic shift exhibit magnetic configurations of the dipole orientations that can strongly differ from those of typical dipolar particles [32]. The capped particles can self-assemble in compact clusters with various magnetic configurations. These configurations can be calculated numerically using sd particles and conducting an energy minimization with respect to the dipole interaction [31]. Here we discuss the configuration of compact clusters made from three and five particles. There exists only one configuration for a three-particle cluster. A ring forms and the caps (or dipoles) form a closed loop [Fig. 1(a)]. For five particles, there also exists only one structural possibility for compact packing. However, the cluster can assume different magnetic configurations. Numerically, we have found several magnetic configurations as local minima of the magnetostatic energy; the four configurations with the lowest energy are 


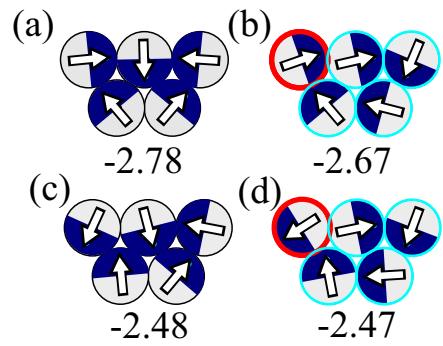

FIG. 6. Clusters of five sd particles $(\xi=0.6)$ with different magnetic configurations. There is only one structural configuration for a compact packing of five particles, but different magnetic configurations exist as local minima. The four states with lowest energy are depicted as configurations (a)-(d), among them (b), (d) the two movers studied experimentally (Fig. 2). White arrows indicate the dipole orientations. The hemisphere with the embedded dipole is dark colored to facilitate visual comparison with the experimental clusters. The numbers below the clusters give the magnetostatic energy per particle, measured in $\frac{\mu_{0} m^{2}}{32 \pi r_{p}^{3}}$.

sketched in Fig. 6. The most stable configuration, A, is the mirror-symmetric extension of the three-membered ring structure, where each side of the cluster represents a closed threeparticle loop. Ising-type frustrated trigonal magnetic lattices exhibit such butterfly-type structure motifs with two neighboring vortices of opposite winding number with increasing temperature [55]. Configuration (b) (Fig. 6) corresponds to the mover depicted in Fig. 2(a), and configuration (d) corresponds to the one in Figs. 2(b) and 2(c). Both are less symmetric than the configuration (a) and slightly less strongly bound. Configuration (b) is also a specific case, because all local moments form a single closed loop; the motion pattern of a cluster with configuration (b) in an external oscillating field thus exhibits strong circular contributions with only a little magnetic anisotropy to direct the translational motion. From a topological point of view, configurations (b) and (d) share the four-particle vortex substructure indicated by blue circles. (b) and (d) only differ in the fact that in (d) the orientation of the upper-left particle (red circle) is reversed with respect to that in B at the expense of $0.2 \frac{\mu_{0} m^{2}}{32 \pi r_{p}^{3}}$. The directed motion depicted in Figs. 2(b) and 2(c) may be understood as the combined rotation of the four-membered subunit indicated by the blue circles and the directed motion of the red-circled particle.

\section{APPENDIX E: HYDRODYNAMIC RADIUS OF AN ACTUATED CAPPED PARTICLE}

In the absence of a dissipative environment, a single dipolar sphere that is exposed to a magnetic torque rotates around its magnetic center. If the magnetic center is shifted away from the center of mass, as given for sd particles, then the rotation leads to a displacement of the sphere center along an arc [Fig. 7(c)] with a radius determined by the shift of the magnetic center. In the presence of a dissipative environment, friction at the particle-fluid interface hinders the rotational and (reversible) translational motion. As a consequence, the particle rotates around the hydrodynamic center of resistance rather than the magnetic center, and the radius of the arc (a)

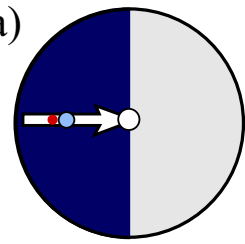

(c)

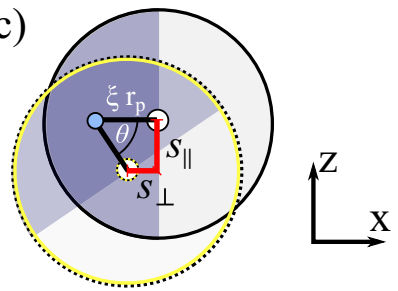

(b)

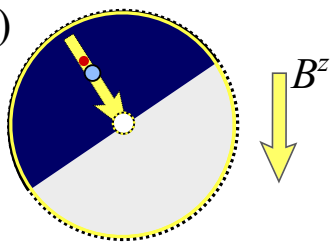

center of mass

- magnetic center

o center of resistance
FIG. 7. Sketch of a sd particle (a) before (solid circle) and (b) after (dotted circle) rotation by $\theta$ caused by $B^{z}$, and (c) an overlay of both situations considering that the particle rotates around the center of resistance that is shifted by $\xi r_{p}$. The center of the sphere moves by $s_{\perp}=\sqrt{x^{2}+y^{2}}\left(\perp B^{z}\right)$ and $s_{\|}\left(\| B^{z}\right)$.

in Fig. 7(c) is determined by the shift $\xi$ of this center of resistance. Brenner's theory for the motion of an arbitrarily shaped particle in a viscous medium can be applied to determine the center of resistance, $z_{R}$ [56-58]. Following Refs. [57,58] for an axis-symmetric anisotropic particle in a viscous environment, the shift $\xi$ of this center of resistance can be calculated from the rotational friction coefficients $f_{t}$ at $z_{R}$ and at the origin $z_{\mathrm{Si}}$ and the origin-invariant translational friction coefficient $f_{t}$ as

$$
z_{R}=\sqrt{\frac{f_{r, \mathrm{Si}}-f_{r, R}}{f_{t}}} .
$$

The rotational friction coefficient around the center of resistance has been determined experimentally as $f_{r, R}=6.5 \times$ $10^{-19} \mathrm{~J} \mathrm{~s}$ [45]. The translational friction coefficient can be calculated from the translational diffusion coefficient $D_{t}$ via the Stokes-Einstein relation $D_{t}=\left\langle\mathbf{r}(t)^{2}\right\rangle / 6 t$. Static, field-free estimates for $D_{t}$ have been obtained by averaging experimentally observed random walk trajectories (Fig. 8). With an experimental value of $D_{t}=0.040 \mu \mathrm{m}^{2} / \mathrm{s}$, the translational friction coefficient amounts to $f_{t}=1.04 \times 10^{-7} \mathrm{~J} \mathrm{~s} / \mathrm{m}^{2}$, which is higher than the ideal value $\left(4.36 \times 10^{-8} \mathrm{~J} \mathrm{~s} / \mathrm{m}^{2}\right)$ calculated from Stokes' law for the rotational friction in an ideally homogeneous medium. This indicates that hydrodynamic interactions of the particles with the substrate enhance the translational friction by a factor of 2.4 .

In contrast to $f_{r, R}$ and $f_{t}$, the rotational friction coefficient around the particle center, $f_{r, \mathrm{Si}}$, is not accessible experimentally in the present setting. It may be obtained from reference data for the silica-water interface via the Stokes equation as $f_{r, \mathrm{Si}}=8 \pi \eta r_{p}^{3}$ [59]. Including the effective increase of the viscosity due to hydrodynamic interactions at the hydrophilic particle-water interface [59], $f_{r, \mathrm{Si}}$ amounts to $7.73 \times 10^{-19} \mathrm{~J} \mathrm{~s}$. Inserting this estimate and the experimental values above in Eq. (E1) gives the center of resistance at $1.07 \mu \mathrm{m}$ from the particle center, which corresponds to a shift $\xi=0.48 r_{p}$. If 
(a)

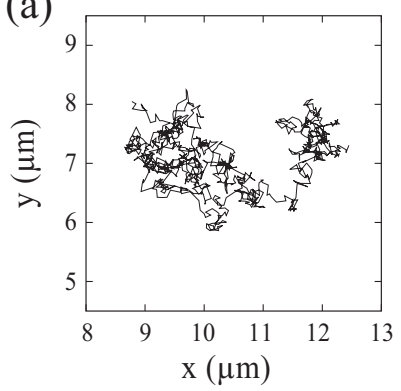

(b)

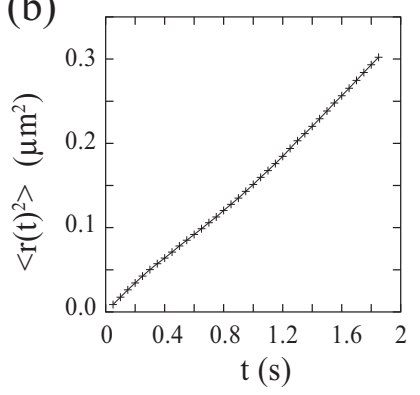

FIG. 8. Translational diffusion of a capped particle with a diameter of $4.54 \times 10^{-6} \mathrm{~m}$ on a glass substrate while the rotational motion is suppressed by an external field. (a) Trajectory of a single particle moving on the $x y$ substrate plane for a time sequence of $20 \mathrm{~s}$. (b) Mean-squared displacement, $\left\langle\mathbf{r}(t)^{2}\right\rangle$, of the trajectory depicted in (a).

substrate effects on the static translational diffusion coefficient $f_{t}$ were negligible in the dynamic regime, the shift would increase to $\xi=0.73 r_{p}$. That value is even higher than the estimated lower bound for the shift of the magnetic center, which is discussed in Appendix A. This shows that within experimental accuracy the center of resistance is located significantly closer to the magnetic center of the particle than to the center of the sphere.

\section{APPENDIX F: ROTATION AND DISPLACEMENT OF A SINGLE SD PARTICLE}

The displacement of a single sd particle in response to an oscillating external magnetic field can be expressed analytically. As indicated in Fig. 7(c), the center of mass of the sphere moves around the center of resistance on an arc with radius $\xi r_{p}$, determined by the magnetic shift and frictional drag. Assuming constant drag, the trajectory of the particle on this arc can be described by the displacements perpendicular $\left(s_{\perp}\right)$ and parallel $\left(s_{\|}\right)$to the oscillating field $B^{z}$ :

$$
s_{\perp}=\xi r_{p}(\cos \theta-1), \quad s_{\|}=\xi r_{p} \sin \theta,
$$

where $\theta$ is the rotation angle [Fig. 7(c)]. For a single particle in a uniaxial field $B^{z}$, the turning points of this trajectory depend on the initial angle $\theta_{0}$ of the Janus director and the $(x, y)$ plane, and the magnitude of the applied external field. If, as presently, the Janus director is parallel to the $(x, y)$ plane in the field-free state, then the turning points are analytically given as a function of $B^{z} / \omega_{B}$, where $\omega_{B}$ is the frequency of the field oscillation [45].

From the differentiation of $s_{\perp}$ and $s_{\|}$with respect to $\theta$, we obtain the infinitesimal displacements

$$
\mathrm{d} s_{\perp}=\mathrm{d} \theta c(\theta) \xi r_{p}(-\sin \theta), \quad \mathrm{d} s_{\|}=\mathrm{d} \theta c(\theta) \xi r_{p} \cos \theta .
$$

An additional term $c(\theta)$ has been incorporated here, which accounts for sources of anisotropic friction that hinder or enhance the infinitesimal displacement. Moreover, the trajectory deviates from the ideal arc shape if an sd particle rotates close to a substrate wall, as depicted in Fig. 7 and detailed next.

To recap, under external actuation fields a magnetic particle solely rotates if the hydrodynamic center of the sphere

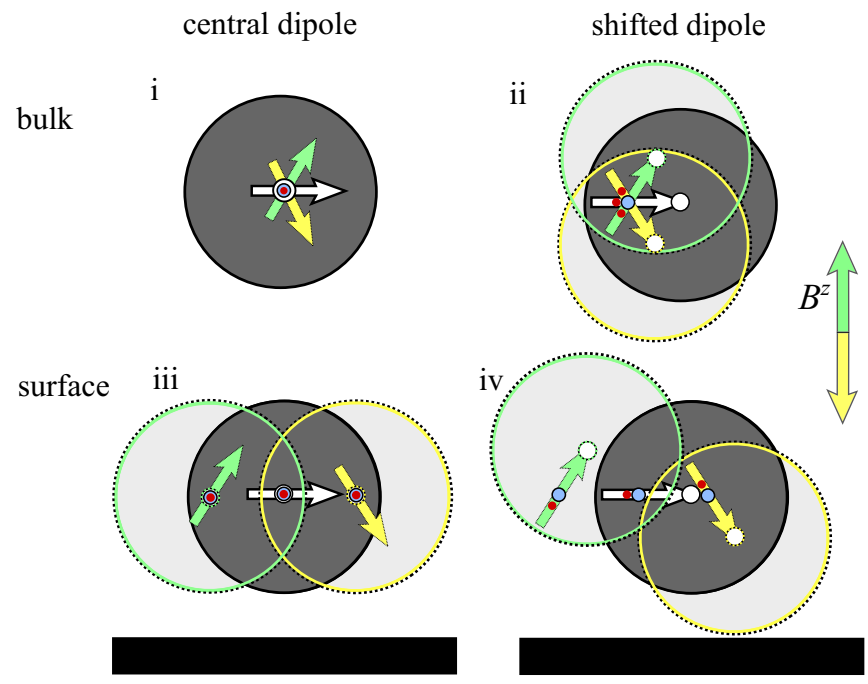

FIG. 9. Rotation of particles with central dipole and sd particles in bulk medium and close to a surface under an external field $B^{z}$. (Same color key as in Fig. 7.)

coincides with its center of mass [case (i), Fig. 9]. Once the hydrodynamic center is shifted, the particle center oscillates along an arc [case (ii), Fig. 9]. The behavior changes in the proximity of a wall, where anisotropic drag is present. For a sphere with central dipole [case (iii), Fig. 9] in a liquid medium, the surface-particle interaction converts rotation into reversible back-and-forth displacement. An sd particle also rolls back and forth, and additionally performs a periodic displacement away from the wall and towards the wall [case (iv), Fig. 9] because the rotational center of resistance is shifted away from the mass center of the sphere. In contrast to case (iii), this motion is not symmetric with respect to $\theta$ due to the hindrance by the wall.

The determination of the hindrance $c(\theta)$ close to a wall [cases (iii) and (iv)] is a complex problem. Since the rotation around the center of resistance periodically changes the distance between the particle center and wall, also the wall-induced hydrodynamic friction coefficient changes periodically. To illustrate the concept, here it is sufficient to approximate the surface-induced drag with a step function of two values $c_{1}, c_{2}$ that alternate with the sign of $\theta$, i.e., whether the dipole points up or down. To distinguish displacement parallel $\left(s_{\|}\right)$and perpendicular $\left(s_{\perp}\right)$ to the actuation, we define

$$
c_{\perp}(\theta)=\left\{\begin{array}{ll}
c_{\perp}, 1 & \text { if } \theta \geqslant 0 \\
c_{\perp, 2} & \text { if } \theta<0
\end{array}, \quad c_{\|}(\theta)=\left\{\begin{array}{ll}
c_{\|}, 1 & \text { if } \theta \geqslant 0 \\
c_{\|, 2} & \text { if } \theta<0
\end{array} .\right.\right.
$$

During $\theta>0$ [green circle in case (iv), Fig. 9] the surfaceinduced translation enhances the translation $s_{\perp}$ caused by the oscillation around the off-center dipole. During $\theta<0$ (yellow circle), the direction of translation when rotating near a wall (iv) is opposite to the translation far away from a wall [case (ii)]. In addition, the distance to the wall changes for forward and backward rolling. Therefore, for oscillations close to a wall (iv) the displacement during $\theta>0$ is always larger than during $\theta<0$ and, thus, $\left|c_{1}\right|>\left|c_{2}\right|$. In case (iv), the overall translation direction during $\theta<0$ (yellow circle) is similar to that of the surface-free case (as depicted in Fig. 7) if the 
influence of the surface dominates over the displacement by the oscillation around the center of resistance, and $c_{\perp, 2}>0$. Otherwise $c_{\perp, 2}<0$ holds. Note that in analogy to the surfacefree case, the field-induced oscillation of a single particle close to the wall is also reversible and leads to no overall displacement.

\section{APPENDIX G: NUMERICAL CALCULATION OF PARTICLE ROTATION IN RING CLUSTER}

The rotational motion of interacting sd particles in a ring cluster exposed to an oscillating field $\mathbf{B}^{z}=\mathbf{B}_{0}^{z} \sin (\omega t)$ has been calculated by numerically solving the equation of rotation. In the simulation, the particles are spatially fixed at their center. This assumption is reasonable when studying the rotation of the particles in the reference system of a cluster that does not change its spatial shape. Assuming sd particles with dipole moment $\mathbf{m}$ (unit vector $\hat{\mathbf{m}}$ ) and stray field $\mathbf{B}^{p}$, the equation of rotation is obtained by balancing the rotational drag, $\tau^{d}$, with the magnetic torques between the sd particles and between sd particles and the field, $\tau^{\mathrm{B}}$. Inertial forces have been neglected on account of the dominating drag forces. Due to the dipole shift, besides the aligning torque $\tau^{p}=\mathbf{m} \times \mathbf{B}^{p}$, also the gradient force $(\mathbf{m} \cdot \nabla) \mathbf{B}^{p}$ between the sd particles is relevant. The latter converts into an effective torque $\tau^{F}$ such that the equation of rotation is given by

$$
\tau^{d}=\tau^{p}+\tau^{F}+\tau^{\mathrm{B}},
$$

which can be written as

$$
f_{r} \dot{\Theta}(t)=\mathbf{m} \times \mathbf{B}^{p}+\xi \hat{\mathbf{m}} \times\left[(\mathbf{m} \cdot \nabla) \mathbf{B}^{p}\right]+\mathbf{m} \times \mathbf{B}^{z},
$$

where $\dot{\Theta}$ is the angular velocity of an object with rotational friction coefficient $f_{r}$ and orientation $\Theta=(\theta, \varphi)$. An ensemble of $n$ particles gives a system of $n$ coupled equations of rotation [Eq. (G1)], which are solved iteratively in a selfconsistent cycle. From the state at time $t$, the orientation $\hat{\mathbf{m}}_{i}$ of particle $i$ at time step $t+1$ is obtained by

$$
\hat{\mathbf{m}}_{i}^{(t+1)}=\operatorname{norm}\left[\hat{\mathbf{m}}_{i}^{(t)}+\frac{1}{f_{\mathrm{r}}}\left[\tau^{p}+\tau^{F}+m_{i} \times\left(B_{0}^{z} \cos (\omega t)\right)\right]\right] .
$$

The function "norm[.]" scales the updated magnetic moments $\mathbf{m}^{(t+1)}$ to retain the fixed magnitude $m s$ of the dipoles. In the calculation, a rotational friction coefficient of $f_{r}=100 \frac{\mu_{0} m^{2}}{32 \pi r_{p}^{3}}$ has been applied. It has the dimension of energy since the numerical time steps $\Delta t=(t+1)-t$ are dimensionless and set to 1 . The angular frequency $\omega$ is also a dimensionless quantity and is set to 0.05 .

The rotational motion $\varphi(\theta(t))$ has been obtained by numerically solving the coupled equations of rotation of the interacting sd particles under an oscillating field $B^{z}$, which points along $\theta=-\pi / 2$ perpendicular to the plane of the ring cluster. For each particle, the periodic variation in $B^{z}$ leads to an oscillatory behavior of $\theta(t)$. It is uniform for all particles, because the motion follows the external field, which is identical for all particles in a ring cluster. Additionally, we observe that the oscillation of $\theta$ involves a periodic change of $\varphi$ for interacting sd particles in a ring, as depicted in Fig. 3(b).

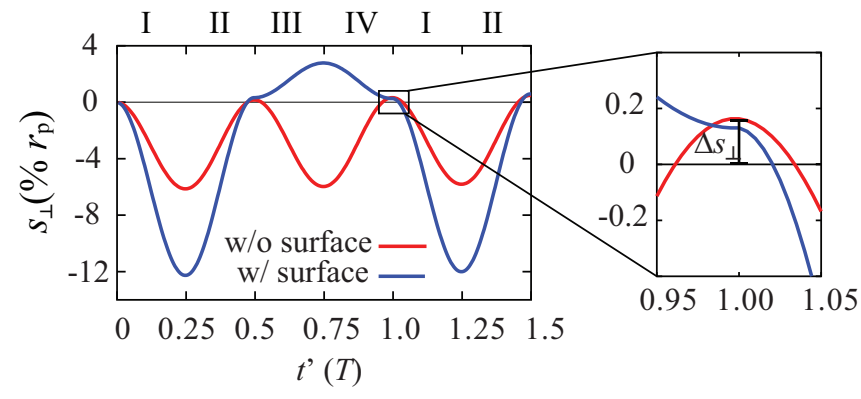

FIG. 10. Translations $s_{\perp}$ of sd particles in a triple ring placed in a homogeneous environment (red curve) and close to a substrate wall (blue curve). The magnified inset on the right illustrates the overall distance a particle has traveled during one field cycle.

\section{APPENDIX H: CALCULATION OF EFFECTIVE PARTICLE TRANSLATION IN A RING CLUSTER}

The time-dependent translation $s(t)$ of sd particles along the orbit of the triple ring can be calculated by integrating Eq. (F2) after inserting analytic expressions for $\theta(\varphi(t))$, and $c(\theta)=\cos [\varphi(\theta(t))]$. The expressions are obtained by fitting the steady-state trajectory $\varphi(\theta)$ [Fig. 3(b)] to a Lissajous curve. The fitting curve has the form

$$
\varphi(\theta)=\varphi_{A} \sin \left(2 \arcsin \frac{ \pm \theta}{\theta_{A}}+p\right)+n,
$$

with the azimuthal and the radial amplitudes $\varphi_{A}$ and $\theta_{A}$, the phase shift $p$ between $\varphi$ and $\theta$, and the offset $n$. The sign \pm accounts for the fact that $\varphi(\theta)$ is a multivalued function. $\varphi$ and $\theta$ perform harmonic oscillations with a frequency ratio of $\omega_{\varphi} / \omega_{\theta}=2$. $\omega_{\theta}$ equals the frequency of the external oscillating field, $\omega_{B}$. With the fit parameters $\varphi_{A}, \theta_{A}, p, n$, the time-dependent torsional oscillations of the particles in a ring can be expressed by $\varphi(t)=\varphi_{A} \sin \left(2 \omega_{B} t+\right.$ $p)+n$ and $\theta(t)=\theta_{A} \sin \left(\omega_{B} t\right)$. From the differentiation $\frac{d \theta(t)}{\mathrm{d} t}$ we obtain the substitution for the integration variable as $d \theta=d t \theta_{A} \omega_{B} \cos \left(\omega_{B} t\right)$. After inserting these expressions into Eq. (1), the time-dependent translation is obtained from the integration $s\left(t^{\prime}\right)=\int_{0}^{t^{\prime}} \mathrm{d} s(t)$. For particles in a ring that is located near a surface, it has to be taken into account that the hindrance term amounts to $c(\theta)=\cos \varphi(\theta(t)) c_{\mathrm{f}}(\theta)$ to account for both the hindrance that neighboring particles exert in each other and the surface-induced friction as sketched in Fig. 9. The effective translation of a particle in the ring is shown in Fig. 10 (blue curve).

If a ring is placed inside an isotropic medium (no wallinduced friction), the overall translation of a particle differs [Fig. 10, red curve]; the sd particles periodically translate back and forth twice per motion cycle [Fig. 9, case (ii)]. Further, we can apply $c_{1}=c_{2}=1$. However, also in this case the particles gain an overall distance $\Delta s_{\perp}$ traveled after each motion cycle $\mathrm{I} \rightarrow \mathrm{IV}$. This demonstrates that the surface is not necessary to induce the actuation presented here; the surface-induced motion alters only the trajectory.

In both cases, $s_{\perp}$ is in the range of a few $\% r_{p}$, and $\Delta s_{\perp}$ is less than one-tenth of that range. The numerical calculation additionally reveals that the particle gains the distance $\Delta s_{\perp}$ with the nonmagnetic side facing forward. Consequentially, 
the uniform translation $s_{\perp}(t)$ of all particles in the ring results in the rotation of the whole ring of the sd particles with the nonmagnetic side facing forward [46]. In order to understand the direction of the overall rotation of the ring, we need to discuss in detail how each part of the rotational motion (I-IV) during the loop contributes to the translational motion. We first discuss the case for a ring in isotropic medium. When the dipole rotates out of the plane (I/III), the particle translates with its magnetic side ahead [Fig. 3(c)], defined as back motion. Forth translation with the nonmagnetic side ahead occurs during Secs. II and IV, i.e., when the dipole rotates back into the plane. As derived from the rotational trajectory [Fig. 3(b)], the factor $\cos \varphi(\theta)$ during I/III is smaller than during II/IV; therefore, $-\mathrm{d} s_{\perp}\left(\theta_{\mathrm{I}}\right)=-\mathrm{d} s_{\perp}\left(\theta_{\mathrm{III}}\right)<\mathrm{d} s_{\perp}\left(\theta_{\mathrm{II}}\right)=$ $\mathrm{d} s_{\perp}\left(\theta_{\mathrm{IV}}\right)$. The integration of $\mathrm{d} s_{\perp}$ over one field cycle is thus positive. The particles in a ring perform a net translation along the orbit with the nonmagnetic side facing forward.

The translation close to a wall requires multiplying Eq. (F2) with the factor $c(\theta)=c_{\mathrm{f}}(\theta) c_{\perp}(\theta)$ and differs qualitatively from the one in an isotropic medium if a value $c_{\perp, 2}<0$ is applied, i.e., if the surface drag dominates over the effect of the magnetic shift on the translation. Then, negative values of $c_{\perp, 2}$ reverse the sign of the displacements in Secs. III and IV of the motion cycle. Thus, forth displacement takes place during II/III and back displacement occurs during I/IV (Fig. 10). During I/II, where $c_{\perp, 1}>0$ is effective, the translation $s_{\perp}(t)$ looks similar to that without a surface. Further, since on the surface $\left|c_{1}\right|>\left|c_{2}\right|, \mathrm{d} s_{\perp}(\mathrm{I})+$ $\mathrm{ds}_{\perp}$ (II) $<\mathrm{ds}_{\perp}$ (III) $+\mathrm{ds}_{\perp}$ (IV) holds. Also, in this scenario the integrated forth displacement $s_{\perp}$ (facing the nonmagnetic side) is larger than the back displacement (magnetic side) during one field cycle. In the example presented in Fig. 10, the ratio $c_{2} / c_{1}=-0.2$ has been applied. This ratio is also visible in the experimental curve $s_{\perp}(t)$ by the ratio between the amplitude during I/II and that during III/IV.

In addition to the translation $\mathrm{d} s_{\perp}$ in the plane of the triple ring, also the motion $\mathrm{d} s_{\|}$parallel to the field (perpendicular to the ring) has to be considered. The particles move collectively up and down, resulting in an oscillating up-and-down translation of the ring. Due to the broken symmetry caused by $\cos \varphi(\theta)$, the trajectory $\mathrm{d} s_{\|}(t)$ is not reversible. However, the vertical component $\mathrm{d} s_{\|}$that is gained during $\mathrm{d} \theta>0$ is reversed during $\mathrm{d} \theta<0$, and the ring gains no overall distance $s_{\|}$.
[1] P. Garstecki and M. Cieplak, J. Phys.: Condens. Matter 21, 200301 (2009).

[2] R. D. Maladen, Y. Ding, C. Li, and D. Goldman, Science 325, 314 (2009).

[3] E. M. Purcell, Am. J. Phys. 45, 3 (1977).

[4] D. A. Fletcher and P. L. Geissler, Annu. Rev. Phys. Chem. 60, 469 (2009).

[5] H. A. Stone and A. D. T. Samuel, Phys. Rev. Lett. 77, 4102 (1996).

[6] R. E. Goldstein, Annu. Rev. Fluid Mech. 47, 343 (2015).

[7] J. Elgeti and G. Gompper, Proc. Natl. Acad. Sci. USA 110, 4470 (2013).

[8] I. Jung, T. R. Powers, and J. M. Valles, Biophys. J. 106, 106 (2014).

[9] E. Lauga and T. R. Powers, Rep. Prog. Phys. 72, 096601 (2009).

[10] C. Bechinger, R. Di Leonardo, H. Löwen, C. Reichhardt, G. Volpe, and G. Volpe, Rev. Mod. Phys. 88, 045006 (2016).

[11] J. Elgeti, R. G. Winkler, and G. Gompper, Rep. Prog. Phys. 78, 056601 (2015).

[12] R. Golestanian, J. M. Yeomans, and N. Uchida, Soft Matter 7, 3074 (2011).

[13] J. J. Abbott, K. E. Peyer, M. C. Lagomarsino, L. Zhang, L. Dong, I. K. Kaliakatsos, and B. J. Nelson, Int. J. Rob. Res. 28, 1434 (2009).

[14] S. J. Ebbens, Curr. Opin. Colloid Interface Sci. 21, 14 (2016).

[15] A. Zöttl and H. Stark, J. Phys.: Condens. Matter 28, 253001 (2016).

[16] J. Palacci, S. Sacanna, A. P. Steinberg, D. J. Pine, and P. M. Chaikin, Science 339, 936 (2013).

[17] A. Erbe, M. Zientara, L. Baraban, C. Kreidler, and P. Leiderer, J. Phys.: Condens. Matter 20, 404215 (2008).

[18] P. J. Vach and D. Faivre, Sci. Rep. 5, 09364 (2015).

[19] P. Tierno, Phys. Chem. Chem. Phys. 16, 23515 (2014).
[20] N. Casic, N. Quintero, R. Alvarez-Nodarse, F. G. Mertens, L. Jibuti, W. Zimmermann, and T. M. Fischer, Phys. Rev. Lett. 110, 168302 (2013).

[21] A. Ghosh and P. Fischer, Nano Lett. 9, 2243 (2009).

[22] A. J. Goldman, R. G. Cox, and H. Brenner, Chem. Eng. Sci. 22, 637 (1967).

[23] P. Tierno, R. Golestanian, I. Pagonabarraga, and F. Sagues, Phys. Rev. Lett. 101, 218304 (2008).

[24] L. Zhang, T. Petit, Y. Lu, B. E. Kratochvil, K. E. Peyer, R. Pei, J. Lou, and B. J. Nelson, ACS Nano 4, 6228 (2010).

[25] P. Tierno and F. Sagues, Eur. Phys. J. E 35, 71 (2012).

[26] R. Trouilloud, T. S. Yu, A. E. Hosoi, and E. Lauga, Phys. Rev. Lett. 101, 048102 (2008).

[27] R. Dreyfus, J. Baudry, M. L. Roper, M. Fermigier, H. A. Stone, and J. Bibette, Nature (London) 437, 862 (2005).

[28] J. J. Benkoski, J. L. Breidenich, O. M. Uy, A. T. Hayes, R. M. Deacon, H. B. Land, J. M. Spicer, P. Y. Keng, and J. Pyun, J. Mater. Chem. 21, 7314 (2011).

[29] E. Gauger and H. Stark, Phys. Rev. E 74, 021907 (2006).

[30] G. Grosjean, G. Lagubeau, A. Darras, M. Hubert, G. Lumay, and N. Vandewalle, Sci. Rep. 5, 16035 (2015).

[31] G. Steinbach, S. Gemming, and A. Erbe, Eur. Phys. J. E 39 (2016).

[32] G. Steinbach, D. Nissen, M. Albrecht, E. V. Novak, P. A. Sanchez, S. S. Kantorovich, S. Gemming, and A. Erbe, Soft Matter 12, 2737 (2016).

[33] P. F. Carcia, A. D. Meinhaldt, and A. Suna, Appl. Phys. Lett. 47, 178 (1985).

[34] M. Albrecht, G. H. Hu, I. L. Guhr, T. C. Ulbrich, J. Boneberg, P. Leiderer, and G. Schatz, Nat. Mater. 4, 203 (2005).

[35] B. Ren, A. Ruditskiy, J. H. Song, and I. Kretzschmar, Langmuir 28, 1149 (2012).

[36] S. K. Smoukov, S. Gangwal, M. Marquez, and O. D. Velev, Soft Matter 5, 1285 (2009). 
[37] J. Yan, S. C. Bae, and S. Granick, Adv. Mater. 27, 874 (2015).

[38] L. Baraban, D. Makarov, M. Albrecht, N. Rivier, P. Leiderer, and A. Erbe, Phys. Rev. E 77, 031407 (2008).

[39] S. Kantorovich, R. Weeber, J. J. Cerda, and C. Holm, Soft Matter 7, 5217 (2011).

[40] See Supplemental Material at http://link.aps.org/supplemental/ 10.1103/PhysRevResearch.2.023092 for movie of the actuated rotation of a triple ring in an oscillating field $\left(B_{0}^{z}=3.25 \mathrm{mT}\right.$, $\left.\omega_{B} / 2 \pi=50 \mathrm{~Hz}\right)$, displayed at 8 times real time.

[41] M. Klinkigt, R. Weeber, S. Kantorovich, and C. Holm, Soft Matter 9, 3535 (2013).

[42] See Supplemental Material at http://link.aps.org/supplemental/ 10.1103/PhysRevResearch.2.023092 for movie of actuated fiveparticle clusters in an oscillating field $\left(B_{0}^{z}=0.50 \mathrm{mT}, \omega_{B} / 2 \pi=\right.$ $5 \mathrm{~Hz}$ ), displayed at 8 times real time. Helical motion switches to directed translation when the field is shortly turned off, which causes particle reorientation in the cluster.

[43] See Supplemental Material at http://link.aps.org/supplemental/ 10.1103/PhysRevResearch.2.023092 for movie of steered, actuated, five-particle clusters in an oscillating field $\left(B_{0}^{z}=1.14 \mathrm{mT}\right.$, $\omega_{B} / 2 \pi=20 \mathrm{~Hz}$ ) overlaid by a constant, weak in-plane field $\left(B^{y}=0.03 \mathrm{mT}\right)$ which aligns the cluster with a significant net magnetic moment. After switching the orientation of the inplane field by $90^{\circ}$, the cluster reorients its translation direction. Movie is displayed at 7 times real time.

[44] C. H. Chen, A. R. Abate, D. Y. Lee, E. M. Terentjev, and D. A. Weitz, Adv. Mater. 21, 3201 (2009).

[45] G. Steinbach, S. Gemming, and A. Erbe, Sci. Rep. 6, 34193 (2016).

[46] See Supplemental Material at http://link.aps.org/supplemental/ 10.1103/PhysRevResearch.2.023092 for a schematic visualiza- tion of the motion pattern of sd particles in a triple ring. First, exclusively the radial motion is shown for three cycles. Following that, the radial motion is combined with the translational motion for two cycles, demonstrating the rotation of the ring.

[47] G. Steinbach, M. Schreiber, D. Nissen, M. Albrecht, E. V. Novak, P. A. Sanchez, S. S. Kantorovich, S. Gemming, and A. Erbe, Phys. Rev. E 100, 012608 (2019).

[48] J. Yan, S. C. Bae, and S. Granick, Soft Matter 11, 147 (2015).

[49] A. B. Yener and S. H. L. Klapp, Soft Matter 12, 2066 (2016).

[50] S. Sacanna, L. Rossi, and D. J. Pine, J. Am. Chem. Soc. 134, 6112 (2012).

[51] A. I. Abrikosov, S. Sacanna, A. P. Philipse, and P. Linse, Soft Matter 9, 8904 (2013).

[52] J. Yan, M. Bloom, S. C. Bae, E. Luijten, and S. Granick, Nature (London) 491, 578 (2012).

[53] F. Kümmel, B. ten Hagen, R. Wittkowski, I. Buttinoni, R. Eichhorn, G. Volpe, H. Löwen, and C. Bechinger, Phys. Rev. Lett. 110, 198302 (2013).

[54] S. C. Glotzer and M. J. Solomon, Nat. Mater. 6, 557 (2007).

[55] T. Kunze, S. Gemming, S. Numazawa, and M. Schreiber, Comput. Phys. Commun. 181, 806 (2010).

[56] H. Brenner, Chem. Eng. Sci. 19, 519 (1964).

[57] J. M. G. Bernal and J. G. De La Torre, Biopolymers 19, 751 (1980).

[58] S. Harvey and J. Garcia de la Torre, Macromolecules 13, 960 (1980).

[59] C. Sendner, D. Horinek, L. Bocquet, and R. R. Netz, Langmuir 25, 10768 (2009). 